\title{
Molecular means and methods
}

Items on view at the meeting of the American Society for Biochemistry and Molecular Biology in San Francisco may include a versatile hybridization oven, affinity purification reagents and a dual-laser-induced fluorescence detector.

Brandel FR115 microfractionator

From Semat

An automatic, programmable clinical fractionator

This microfractionator should provide the precise amounts of solution that need to be extracted from centrifugally banded solutions for clinical analysis. It can collect aliquots as small as $0.25 \mu \mathrm{l}$ from centrifuge tubes without mixing the tube's contents. Applications of this technology include DNA fractioning of LipoProdines, or the quantitative characterization of macromolecules. The FR115 will accept standard cylindrical $1.0-\mathrm{ml}$ and $0.2-\mathrm{ml}$ polycarbonate centrifuge tubes, as well as $0.15-\mathrm{ml}$ capacity, high-precision quartz tubes. In addition, a recently introduced block assembly will accept the 5.1-ml, TLA 100.4 tube used with Beckman's rotor TL100 machines, and is interchangeable with the supplied assembly. The use of an open design in the FR115 allows adaptations for special applications and additional tube types to be incorporated easily. The size and number of fractions, the collection rate and all other variables are user adjustable through the programmable keypad. Individual fractions can be collected in liquid form, with or without chase or scintillation fluid, using an outlet capillary, or they can be deposited directly onto glass-fibre filters. The instrument can be employed manually in single-step operation, using a panel button or foot pedal, or automatically in a repetitive mode, using an external pump and fraction collector.

Reader Enquiry No. 100

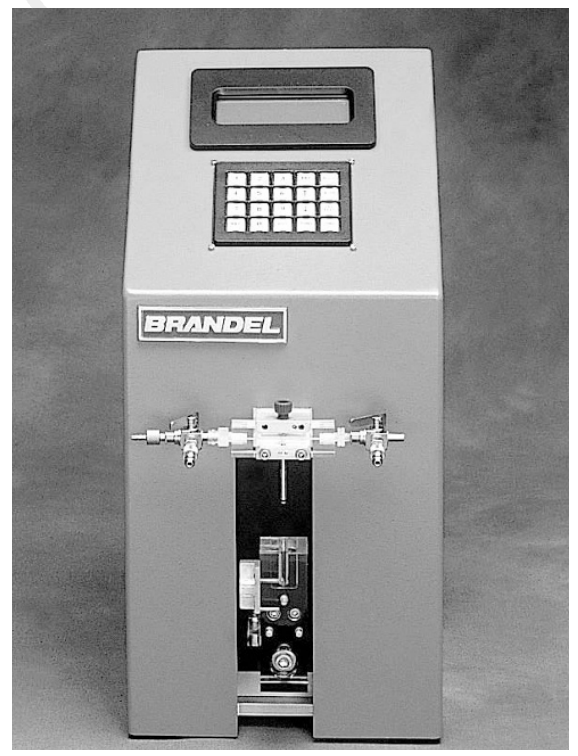

The FR115 automatic microfractionator.

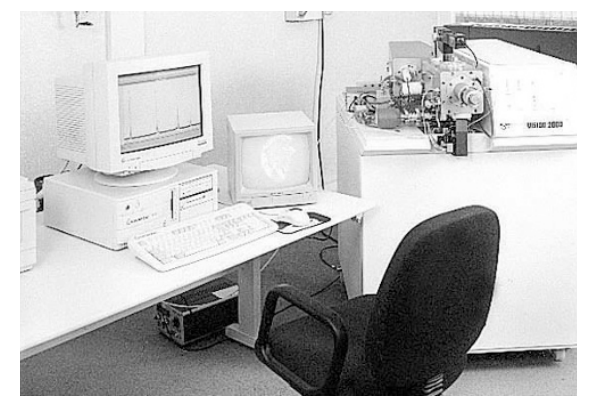

Vision 2000 MALDI MS for DNA sequencing.

Vision 2000

From Thermo BioAnalysis

A system for DNA sequencing that uses MALDI mass spectrometry

According to the manufacturer, this spectrometer has been used to read DNA sequencing ladders generated by solid-phase Sanger sequencing protocols. Conventional DNA sequencing using Sanger chemistry employs gel electrophoresis to separate specifically terminated DNA fragments within the four-base sets. In methods that use MALDI MS, DNA is immobilized onto magnetic beads using biotin-streptavidin chemistry. After sequence ladder generation, the beads are placed directly onto a Vision 2000 sample target for analysis. The methods, developed by Hubert Köster, are said to demonstrate the potential for increasing throughput in DNA sequencing by several orders of magnitude with improved accuracy, reliability and higher base identification.

Reader Enquiry No. 101

\section{Spin'n'Stack oven}

From Hybaid

For whole-mount in situ hybridization

The 'tilting wheel' accessory provided with the oven enables whole-mount samples to be rotated at speeds between 5-20 r.p.m. and at adjustable tilt angles between $0^{\circ}$ and $180^{\circ}$. These two provisions allow for the use of minimal solution volumes and provide the gentle mixing action required for fragile whole-mount samples. In conjunction with the accurate temperature control and uniformity provided by the oven, suitable conditions can be created for whole-mount in situ protocols. Tube carousels are available to hold a variety of tube types from $0.5-\mathrm{ml}$ microcentrifuge tubes through to 50 - $\mathrm{ml}$ tissue culture tubes. These double as tube racks to enable easy benchtop manipulations and sample storage. The oven has been designed to be fully compatible with the existing Shake'n'Stack oven accessories, so that a range of hybridization rotisseries and a shaking platform can be used if desired.

Reader Enquiry No. 102

\section{Gels analysis and separation \\ Hi-Lo DNA marker \\ From BioNexus \\ A marker that covers the range from $50 \mathrm{bp}$ to $10,000 \mathrm{bp}$}

According to the manufacturer, the allpurpose Hi-Lo DNA marker is easy to read with several landmarks and logically spaced bands. The marker is informative, allowing accurate sizing of DNA bands over its entire range. This is said to eliminate the need to purchase two separate markers. It is stable at room temperature, allowing shipping and storage at ambient temperature. Reader Enquiry No. 103

\section{Speedlight Platinum GDS}

From Lightools Research

A workstation designed from the ground up for gel documentation

This system incorporates on-chip integration for very low light level imaging, digital storage to disk for long-term archival and post processing, time/date/ID and text for annotation, as well as digital background subtraction, sensor lock for 'sharp' prints, and camera saturation detection to keep images in the camera's linear dynamic range. Users can access all of these functions, according to the manufacturer, through a rugged, hand-held controller. The design philosophy is for centralized gel documentation and off-line, decentralized

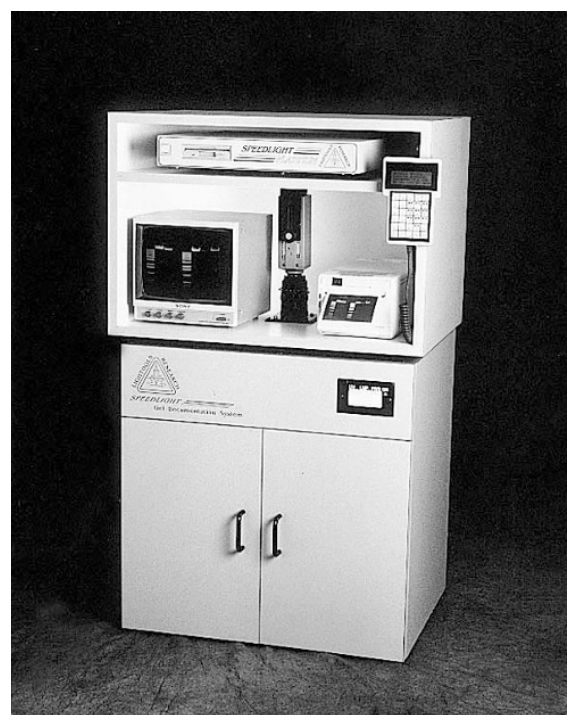

A gel documentation system, Lightools Research. 
data processing to eliminate the gel documentation system from becoming a data processing bottleneck.

Reader Enquiry No. 104

\section{EKAway}

From Invitrogen

Enterokinase removal using affinity resin

Many protein purification strategies incorporate affinity tags for purification by affinity chromatography. Of these, a number also contain enterokinase cleavage sites for the removal of the affinity tag by treatment with enterokinases, for example EKMax. However, in order to obtain high-purity recombinant protein it is often necessary to remove the contaminating enterokinase itself. EKAway is an enterokinase affinity resin designed specifically for this purpose and is stated to remove greater than 99 per cent of the enterokinase from the protein/ enterokinase mixture. It is also said to be effective in removing other trypsin proteases. Reader Enquiry No. 105

\section{Oligo-dT 30 particles}

From Scigen

A range of affinity purification reagents, including paramagnetic oligo- $d T_{30}$ particles for rapid $m R N A$ separation

The magnetic Oligo- $\mathrm{dT}_{30}$ particles are said to be well suited for isolating mature eukaryotic mRNA from crude RNA preparations or cell lysis extracts for a cost that is stated to be 3-7 times less than other oligo-dT particles. Each Oligo- $\mathrm{dT}_{30}$ is covalently coupled via a $5^{\prime}$ spacer to enhance specific binding at high stringency and minimize steric hindrance. The density of the 3- $\mu \mathrm{m}$ particle has been optimized for mRNA isolation, providing easy mixing with fast magnetic sedimentation.

\section{Reader Enquiry No. 106}

\section{1 image analysis software}

From Eastman Kodak

Version 2.0 of the company's onedimensional image analysis software for Macintosh and Windows

The software acquires digital images using Kodak's Digital Science cameras or any TWAIN compliant scanner, and is well suited for analysing DNA, RNA and protein electrophoresis gels and blots. Analysis data includes mass, molecular weight, intensity and mobility values. This new version offers users several new features, including automated lane finding, non-destructive annotations, Gaussian band fitting, enhanced printing capabilities and support for Kodak's newly released digital DC120 camera. The software allows users to annotate their images for documentation and presentation purposes. This object-oriented

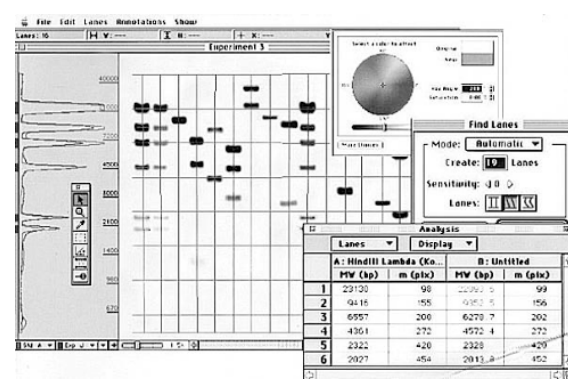

Kodak's 1D image analysis software version 2.0.

layer supports a variety of custom lines, arrows and assorted shapes of any colour. Additionally, users have the ability to crop their image or create custom views of the images. A Gaussian band-finding algorithm has been incorporated into the software, which is specifically designed to improve analysis data for saturated bands, unresolved bands, or for the analysis of images with uneven backgrounds. Version 2.0 offers flexibility in printing, allowing data to be output in any one of over 16 different formats. With the 'summary' option, a user can print a single-page summary that includes the gel image, all lane information, and the analysis results for one variable of interest. In version 2.0, integrated support for Kodak's new DC120 megapixel camera is available. This new high-resolution camera offers high-quality images for the direct capture of ethidium bromide and protein gels.

Reader Enquiry No. 107

\section{Wide Mini Poly (NAT) gels}

From Guest Elchrom Scientific

High-resolution, high-throughputprecast submarine gels

These gels are intended for the analysis of DNA fragments in those applications that require high resolution and high throughput. The gels are produced in two formats and in three concentrations to cover the size range from 10 to $20,000 \mathrm{bp}$. Size differences of 1-2 per cent can be detected in the range between 60 and 2,000 bp on Wide Mini S-50 gels, which have a running path of $8.7 \mathrm{~cm}$ and contain 50 sample wells. For $\mathrm{S}-100$ gels, the running path is $4.0 \mathrm{~cm}$.

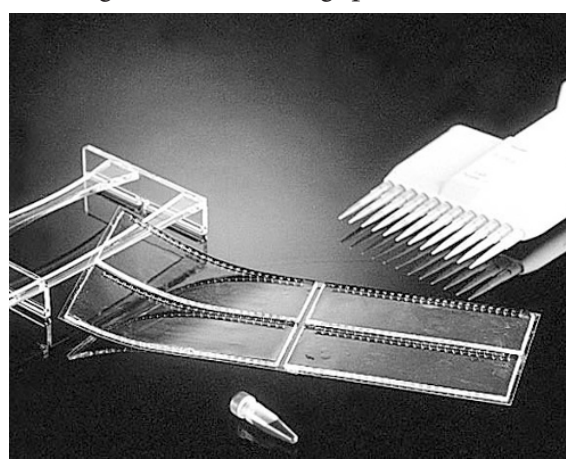

Guest Elchrom Scientific's wide precast gels.
Moreover, both S-100 and S-50 gels consist of separate 25-well sections, which can be run independently after cutting off the plastic backing to which the gel is covalently linked. Sample loading is fast with a 12-channel pipettor. The gels can be run in most existing submarine units but the improved results are often obtained when running them in Elchrom's SEA 2000, which allows the analysis of more than 500 samples per day and is said to lower cost substantially over some precast mini gels. These gels are well suited for analysing amplified DNA fragments, such as RAPD, STR and heteroduplexes.

Reader Enquiry No. 108

\section{Usingradioactivity}

RaylMax

From ICN

A new product line that should complement the company's radiochemical and non-isotopic labelling reagents

ICN has three types of the clear-based RayMax autoradiography film that should enable researchers to choose a film to suit the application. RayMax Universal is a doublecoated, multipurpose film for general autoradiography and fluorography, offering high-resolution results at economical prices. The film consists of a clear plastic base, coated on both sides with a photographic emulsion. The emulsion is coated with a protective anti-scratch layer. This film is recommended for a multi-disciplinary laboratory where it will give high performance in a variety of applications. RayMax Beta is a single-coated, fine-grain emulsion film that gives high resolution in direct autoradiography of ${ }^{125} \mathrm{I},{ }^{33} \mathrm{P},{ }^{35} \mathrm{~S}$ or ${ }^{14} \mathrm{C}$. It has a high silver content that is said to increase the absorption efficiency, resulting in high-speed exposures. RayMax Tritium is a single-coated film without an anti-scratch layer, which allows for direct autoradiography of tritium or ${ }^{125}$ I. This film offers the high resolution for the detection of very weak $\beta$ particles of ${ }^{3} \mathrm{H}$ or lowenergy Auger electrons associated with ${ }^{125} \mathrm{I}$ decay. Each type of film is available in a range of sizes to serve various applications

Reader Enquiry No. 109

$\beta$ and $\gamma$ radiation products

From Ultra Violet Products

A range that includes cabinets, shields, boxes and bins

All of the products in this line are manufactured from material that effectively blocks out the most often used $\beta$ isotopes of ${ }^{32} \mathrm{P}$, ${ }^{35} \mathrm{~S},{ }^{3} \mathrm{H}$ and ${ }^{14} \mathrm{C}$, and the $\gamma$-isotopes of ${ }^{125} \mathrm{I}$. The beta and gamma cabinet has been designed as a fully enclosed workstation with large access doors and a good sized working space. It can be placed on a range of laboratory trays, and when the doors are 


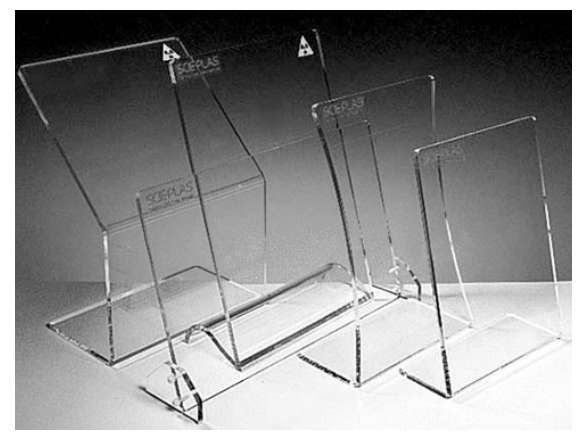

UVP offers products for working with beta and gamma radiation.

closed, allows radioactive materials to be safely isolated. There is also a large selection of shields available in various styles and sizes, depending on the requirements of the user. The company can also provide boxes for the storage of various sizes of sample containers. With interchangeable inserts, these boxes are suited for storing Eppendorf tubes, centrifuge tubes, Falcon tubes, scintillation vials and cryotubes. There are also nine different size bins for radioactive waste.

\section{Reader Enquiry No. 110}

\section{DNA detection and analysis}

MAP kinase SPA

From Amersham Life Science

A new SPA assay for MAP kinase activity that is designed to save time in direct quantification

It is now possible to quantify directly mitogen-activated protein kinase (MAPK) activity in a high-throughput format. This follows the company's launch of MAP kinase SPA ${ }^{33} \mathrm{P}$. Not only does the new assay offer an insight into understanding important signal transduction pathways, it is also said to be less time-consuming than conventional assays (which usually involve the capture, separation and washing of radiolabelled, phosphorylated peptides). MAPK is an important component of many signal transduction pathways, activating a multitude of intracellular molecules. The singletube homogeneous format enables the assay to be performed in 96-well microplates and is therefore amenable to automation and high-throughput screening applications. Developed using the enzyme ERK-1, the new assay also has the potential to be used with other MAP kinases, in particular, p38

Concept for the MAP Kinase SPA assay

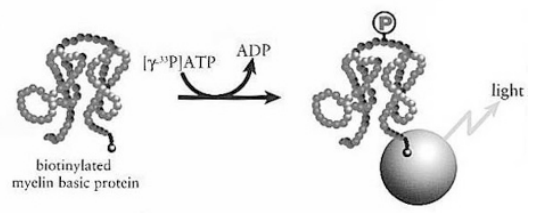

Amersham MAPK quantification assay. and JNK. The assay relies on the MAPK-catalysed transfer of the $\gamma$-phosphate group of $\gamma-{ }^{33} \mathrm{P}$-ATP to a 'common' substrate (biotinylated myelin basic protein, bMBP). The radiolabelled, biotinylated product is then captured onto streptavidincoated SPA beads, bringing the label close enough to the scintillant to generate a light signal. Sufficient reagents are included to perform 500 assays.

Reader Enquiry No. 111

\section{Transcend}

From Promega

A chemiluminscent translation detection system that allows the non-radioactive detection of proteins translated in vivo

Promega states that this system offers a safer, more convenient alternative to isotopes and suffers no loss in sensitivity. The key element in these systems is the tRNA, a biotinylated lysyl tRNA. When added to an in vitro translation reaction the biotinylated lysine is incorporated into the newly translated protein. The protein can then be detected with streptavidin-horseradish peroxidase and Transcend chemiluminescent substrates. The chemiluminescent detection reagents are based on luminol and are said to be well suited for the detection of proteins on nitrocellulose or PVDF membranes. As lysine is more prevalent than the commonly used radiolabelled amino acid methionine, this system should prove useful for those studying proteins with few methionines or truncated proteins, as in the protein truncation test (PTT). Biotinylating the protein not only allows for its nonradioactive detection, but also for its capture either on streptavidin resins or membranes. A colorimetric detection alternative is also available.

Reader Enquiry No. 112

\section{DUSeries 500 spectrophotometers}

From Beckman Instruments

Ultraviolet/visible spectrometers are designed to offer affordability for routine applications

These new spectrophotometers are said to combine easy sample handling with accurate measurement and interchangeable sampling modules that allow the user to reconfigure the system quickly and easily. Each system comes with a complete set of application programs. There are two models available: the DU 520, which delivers accurate measurements for applications such as single and multiple wavelength analysis, wavelength scanning, spectral manipulation, time-based kinetic/rate determination and single-component analysis for concentration determination, and the DU 530, which offers all these features plus application programs for protein and nucleic acid analysis, including DNA/RNA/oligonucleo-

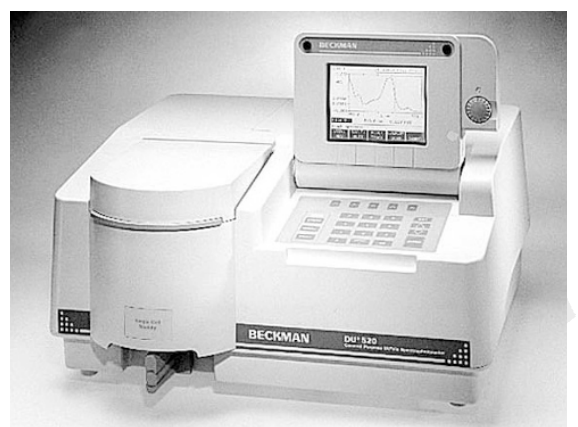

Beckman's DU series 500 spectrometers.

tide quantification modes for determining molecular weight, concentrations and theoretical melting point.

Reader Enquiry No. 113

\section{DNAsis}

From Hitachi Software

Version 2.5 of this software is offered, as are upgrade and demonstration CDs

This newly upgraded gene analysis software program includes a direct interface to the NCBI BLAST database. Searches can be initiated within DNAsis and results opened in either a text file or as an HTML file. Primer design functions have also been added, including dimer, self-dimer, hairpin, repeat and false-priming site analysis. This version also includes a file converter, so that data may be shared between laboratories that have different DNA sequence analysis packages. All new upgrade features can be saved and printed, or imported to a favourite word processing or similar package for further manipulation. A free demonstration CD is available for this software package, which includes ScanDNAsis, an electrophoresis gel image analysis software for Macintosh computers; DNAsis for Windows and MacDNAsis provide full range sequence analysis software packages; and HybSimulator allows primer design and simulation for Macintosh or Windows platforms.

Reader Enquiry No. 114

\section{BioFocus LIFF²}

From Bio-Rad

A dual-laser-induced fluorescence detector

The new BioFocus LIF $^{2}$ detector for capillary electrophoresis has dual detection and improved sensitivity. Because the detector allows excitation with two separate, highintensity lasers and simultaneous dual detection of emitted fluorescence, it provides much greater flexibility and allows higher productivity, as the unknown sample and the standard can be run at the same time for optimal internal referencing. Stated to be 1,000 times more sensitive than conventional ultraviolet absorbance detectors, this detector can detect picomo- 


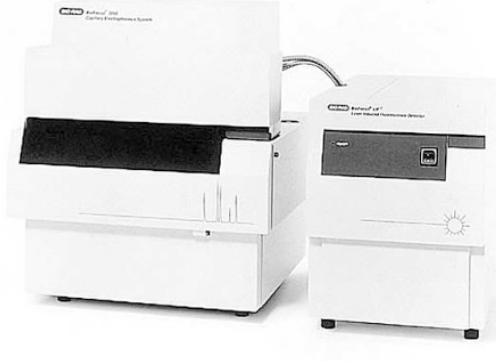

The BioFocus LIF ${ }^{2}$ dual-laser-induced fluorescence detector from Bio-Rad.

lar concentrations of DNA. The system is available in both single- and dual-laser configurations, and is compatible with a range of external lasers to meet specific application requirements.

Reader Enquiry No. 115

\section{Anti-Lewis ${ }^{y}$ monoclonal antibody}

From Dako

A monoclonal antibody that should assist in apoptosis detection

$\mathrm{Le}^{\mathrm{y}}$ is an oligosaccharide connected with other Lewis substances: $\mathrm{Le}^{\mathrm{a}}, \mathrm{Le}^{\mathrm{b}}$ and $\mathrm{Le}^{\mathrm{x}}$. Although its function is not fully understood, $\mathrm{Le}^{\mathrm{y}}$ has been identified phenotypically as a marker of specific cell types and possibly of specific stages of cell differentiation. Recently, $\mathrm{Le}^{\mathrm{y}}$ was found to be a marker characteristically present on cells undergoing apoptosis. There is said to be a good correlation between $\mathrm{Le}^{\mathrm{y}}$ expression and DNA fragmentation, as measured by $3^{\prime}$-OH-end labelling. There is no expression of $\mathrm{Le}^{\mathrm{y}}$ on cells undergoing differentiation or necrosis. The new monoclonal antibody is derived from clone BM1, which is said to work well in formalin-fixed, paraffin-embedded tissue sections, and should be useful in studies of the apoptotic process. A large number of other antibodies connected with apoptosis, including anti-bcl-2 and anti-p53, are also available from Dako.

Reader Enquiry No. 116

XL10-Gold ultracompetent cells

From Stratagene

Plasmid libraries that are now said to approach the quality of lambda libraries

Statagene states that these new cells were derived to provide the highest transformation efficiencies available for ligated DNA, stated to produce up to 25 -fold more primary clones than DH10B cells. Plasmid libraries constructed in XL10-Gold cells may prove to be larger and more complex, approaching the quality of lambda libraries. Stratagene has developed three plasmid/cDNA synthesis kits: the pBluescript II XR library construction kit for prokaryotic cloning, the pCMV-Script XR library construction kit for high-level eukaryotic expression and the pADGAL42.1 XR library construction kit for two-hybrid screening of yeast. All three kits are provided with sufficient reagents for five cDNA synthesis reactions, a predigested vector and XL10-Gold ultracompetent cells. Each kit is quality controlled to produce $1 \times 10^{6}$ primary clones with test mRNA.

Reader Enquiry No. 117

\section{TransIT}

From PanVera

Reagents for efficient DNA and RNA transfections

These reagents are said to offer economical, high-efficiency transfection with minimal cellular toxicity in both stable and transient transfections. The products also offer reproducibility, long shelf life and straightforward optimization. This combination of features makes TransIT reagents well suited for all gene expression studies where the post-transfection state of the cell is important. TransIT reagents

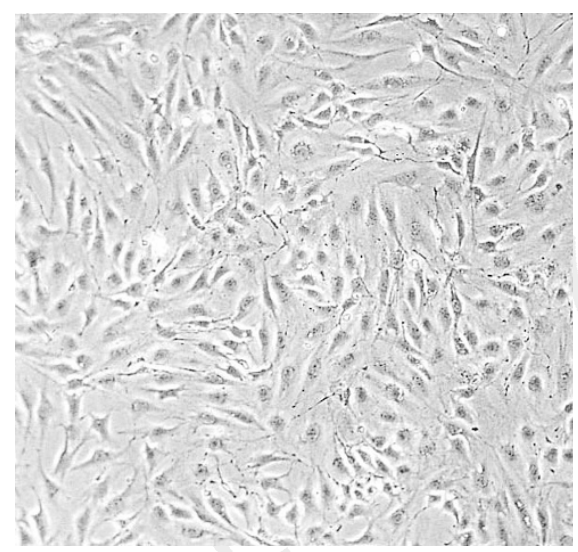

PanVera's TransIT reagents for high-throughput screening or high volume protein production.

can also be used for high-throughput transfection screening or high-volume protein production.

Reader Enquiry No. 118

\section{RetroXpress}

From Clontech

A new retroviral gene expression system

RetroXpress has been formulated to accomplish efficient retrovirus-mediated gene transfer and expression. The kit includes the RetroPack PT67 packaging cell line and RetroXpress vectors for introducing genes into a wide variety of mammalian cell types (in vitro or in vivo). The PT67 packaging cell line contains stably integrated retroviral structural genes for the safe production of high titres of infectious, replication-incompetent retroviruses. Transduction of the recombinant retroviral particles is said to result in nearly 100 per cent of cells expressing the gene of interest.

Reader Enquiry No. 119

These notes are compiled by Brendan Horton from information provided by the manufacturers. For more details, fill in the reader service card bound inside the journal.

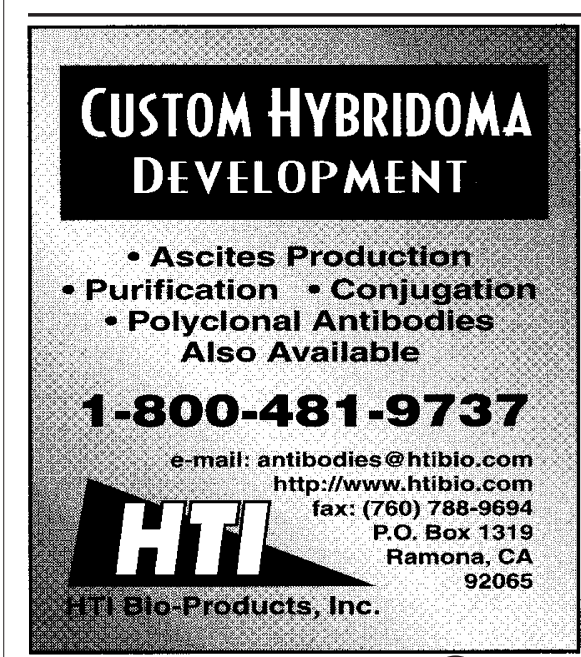

READER ENQUIRY NO. 96

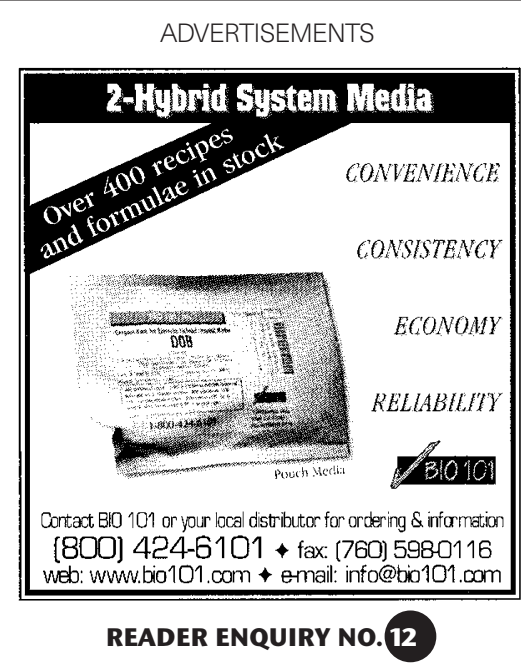

Nature @ Macmillan Publishers Ltd 1997

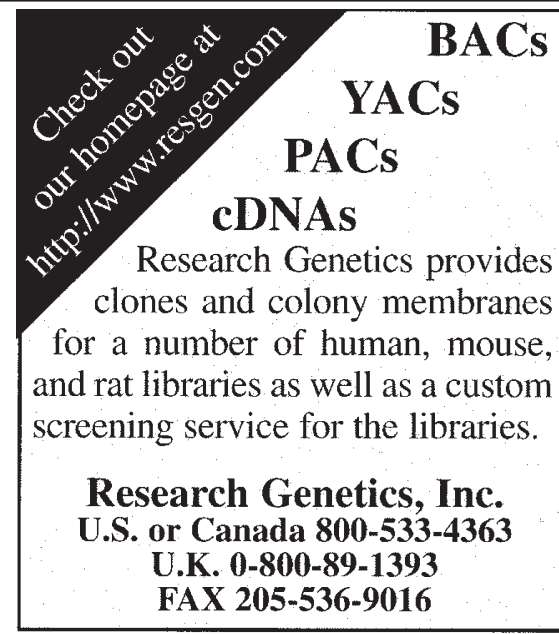

READER ENQUIRY NO. 92

NATURE | VOL 388 | 21 AUGUST 1997 